\title{
Robustness of Multiplicative Processes in Auditory Spatial Tuning
}

\author{
José Luis Peña and Masakazu Konishi \\ Division of Biology 216-76, California Institute of Technology, Pasadena, California 91125
}

\begin{abstract}
Auditory space-specific neurons in the owl's inferior colliculus selectively respond to the direction of sound propagation, which is defined by combinations of interaural time (ITD) and level (ILD) differences. Mathematical analyses show that the amplitude of postsynaptic potentials in these neurons is a product of two components that vary with either ITD or ILD. Temporal correlation in the fine structure of signals between the ears is essential for detection of ITD. By varying the degree of binaural correlation, we could accurately change the amplitude of the ITD component of postsynaptic potentials in the space-specific neurons. Multiplication worked for the entire range of postsynaptic potentials created by manipulation of ITD.
\end{abstract}

Key words: auditory; binaural; sound localization; multiplication; barn owl; interaural correlation

\section{Introduction}

Multiplication is an important operation in models of the nervous system (Stern and Colburn, 1978; Poggio, 1990; Salinas and Abbott, 1996; Pouget and Sejnowski, 1997; Koch, 1999). However, few experimental studies have demonstrated its use by neurons (Egelhaaf et al., 1989; Hatsopoulos et al., 1995; Andersen et al., 1997; Sun and Frost, 1998; Peña and Konishi, 2001; Chance et al., 2002; Gabbiani et al., 2002). Multiplication makes possible the implementation of nonlinearities such as those required for neuronal selectivity for combinations of stimulus features. No studies have asked whether this mathematical operation is independent of the range and strength of the input. The owl's auditory system provides a cell group in which we can examine the above question. Barn owls use differences in interaural time (ITD) and level (ILD) to localize sound sources in the horizontal and vertical directions, respectively. These binaural cues are processed in separate neural pathways that emerge from the cochlear nuclei and converge on the inferior colliculus (Takahashi et al., 1984). The space-specific neurons of the external nucleus of the owl's inferior colliculus (ICx) selectively respond to particular combinations of ITD and ILD (Moiseff and Konishi, 1983; Olson et al., 1989). We showed previously that the amplitude of postsynaptic potentials (PSPs) for different ITD-ILD pairs is a product of two independent components; one varies with ITD and the other with ILD (Peña and Konishi, 2001). The present paper tests how the multiplicative process works when one input is systematically changed.

Received July 19, 2004; revised Sept. 1, 2004; accepted Sept. 3, 2004.

This work was supported by a grant from the National Institute of Neurological Disorders. We thank Bjorn Christianson and Sharad Shanbhag for their comments on previous drafts; Partha P. Mitra and Fabrizio Gabianni for their advice in using SVD; and Gilles Laurent, Christof Koch, and Bartlett Mel for reviewing drafts of this manuscript.

Correspondence should be addressed to José Luis Peña, Division of Biology 216-76, California Institute of Technology, Pasadena, CA 91125.E-mail: jose@etho.caltech.edu.

DOI:10.1523/JNEUROSCI.2924-04.2004

Copyright $\odot 2004$ Society for Neuroscience $\quad$ 0270-6474/04/248907-04\$15.00/0

\section{Materials and Methods}

Owls were anesthetized by intramuscular injection of ketamine hydrochloride (25 mg/kg; Ketaset; Phoenix Pharmaceuticals, Mountain View, CA) and diazepam (1.3 mg/kg; Steris Laboratories, Phoenix, AZ). The protocol for this study followed the National Institutes of Health Guide for the Care and Use of Laboratory Animals and was approved by the Institute Animal Care and Use Committee. The data came from in vivo intracellular recording of 26 space-specific neurons obtained in five owls.

Acoustic stimuli were digitally synthesized with a personal computer and delivered to both ears by calibrated earphone assemblies. Auditory stimuli consisted of broadband noise bursts $[0.5-12.0 \mathrm{kHz} ; 50$ or 100 $\mathrm{msec}$ in duration and $5 \mathrm{msec}$ rise and decay times; sound level of $40-50$ $\mathrm{db}$ sound pressure level (SPL)]. The computer synthesized three random noises to obtain different values of binaural correlation. One of them was delivered to one ear and its copy to the other ear, making the correlated component of the stimulus. Each of the other two noises was used as the uncorrelated component of the stimulus by adding it to the correlated noise while keeping the sound level constant. Binaural correlation (BC) varies with the relative amplitude of the uncorrelated and correlated noises by $\left(1 /\left(1+k^{2}\right)\right)^{1 / 2}$, where $k$ is the ratio between the root-meansquare amplitudes of the uncorrelated and correlated noises.

We computed the median membrane potential during the first 50 msec of the response to sound and averaged it over three to five stimulus presentations (Peña and Konishi, 2001). The data were arranged in matrices that contained the response in PSP amplitude for different combinations of ITD and ILD. We used singular value decomposition (SVD) to analyze these data. This method transforms matrices into a weighted sum of products of independent vectors, called "singular vectors." The contribution of these products to the original matrix is ranked by the elements ("singular values") of a diagonal matrix. If the first product constitutes all or most of the data matrix, it can be deduced that the data are derived from a multiplication of the first set of singular vectors. The membrane potentials in response to ITD-ILD pairs were arranged in a matrix in which ILD and ITD varied along rows and columns, respectively. We assume that each matrix $(M)$ contains constant and multiplicative components as in the following equation: $M(i, j)=V_{0}+$ $\lambda \mathbf{U}(i) \mathbf{V}(j)^{\mathrm{T}}$, where $V$ o represents a membrane potential offset. The second term indicates the multiplicative interaction between ITD and ILD inputs, $\mathbf{U}(i)$ and transposed $\mathbf{V}(j)$, and $\lambda$ is a singular value. We deter- 
mined the optimal Vo value in the equation by minimizing the sum of the squared singular values other than the first one in the SVD of $M-V$ o. This procedure is comparable with a least-square fit to the multiplicative model with one adjustable variable ( $V o$ ). After subtracting $V o$, we calculated the relative weight of the first singular value as the square of this value divided by the sum of the squares of all singular values. This ratio shows the contribution to the data matrix of a first-order relationship (multiplication) between the two vectors $\mathbf{U}_{1}$ and $\mathbf{V}_{1}$. These vectors yield functions similar to the measured ITD and ILD response curves of the neuron (Peña and Konishi, 2001).

A pure additive combination of ITD and ILD inputs can be given by the following: $M(i, j)=V_{0}+\mathbf{F}(i)+\mathbf{G}(j)$, where $V_{0}$ is a constant, and $\mathbf{F}(i)$ and $\mathbf{G}(j)$ are the ITD and ILD inputs. All values were obtained by a least-square fit of the data. The initial values of $V_{0}, \mathbf{F}(i)$, and $\mathbf{G}(j)$ were the mean membrane potential, the average across rows, and the average across columns of the data matrix, respectively. The fitting algorithm was set to independently vary the initial values of $V o$ and each element of the input vectors to minimize the difference between the results and the data. We used an additive model with the maximum number of variables (degrees of freedom) to compare the results obtained by SVD with the best possible representation of the data by addition.

\section{Results}

Birds and mammals use a process similar to cross-correlation to detect ITDs (Licklider, 1959; Carr and Konishi, 1990; Yin and Chan, 1990). This computation relies on the similarity between the signals in the left and right ears (i.e., the BC). Addition of random noise to correlated signals produces different degrees of BC (Jeffress and Robinson, 1962; Albeck and Konishi, 1995, Saberi et al., 1998). Changes in BC vary the signal-to-noise ratio in the temporal domain of the stimulus without affecting its amplitude. Thus, we can independently vary the ITD input to spacespecific neurons while we perform intracellular recording.

When the stimulus is broadband, the membrane potential of space-specific neurons shows a depolarizing peak at a particular ITD $\left(\operatorname{ITD}_{\mathrm{m}}\right)$ and smaller "side peaks" at $\operatorname{ITD}_{\mathrm{m}} \pm n T$, where $T$ is the period of the best frequency of the neuron, and $n$ is an integer. Both the depolarizing and hyperpolarizing deflections of the membrane potential in response to ITD became smaller when BC was reduced (Fig. 1a). Although the amplitude of ITD curves varied as a function of $\mathrm{BC}$, neither the tuning nor the amplitude of ILD curves changed when BC was varied from 0 to 1 (Fig. $1 b$ ). The depolarizing input to the neurons decreased as $\mathrm{BC}$ declined, although sound level remained constant (Fig. 1c,d).

We used the methods of SVD to analyze the data (see Materials and Methods). We performed the SVD on subthreshold PSPs obtained for different combinations of ITD and ILD in 15 spacespecific neurons, an example of which is shown in Figure $2 a, b$. The amplitudes of ITD singular vectors changed for different BCs (Fig. 2a), whereas those of ILD vectors remained essentially unchanged (Fig. $2 b$ ). For the entire sample, the first singular values carried $96.0 \pm 0.8 \%$ of the weight for all values of BC (Fig. $2 c$ ), indicating that despite changes in $\mathrm{BC}$, the subthreshold receptive fields are always a product of two vectors.

However, a high first singular value is consistent with multiplication of the ITD and ILD inputs only if the vectors to be multiplied are correlated with the tuning to ITD and ILD. We therefore analyzed the structure of the first singular vectors when $\mathrm{BC}$ was changed. Both the amplitude and shape of ILD vectors remained unchanged with $\mathrm{BC}$, showing that the tuning to ILD is immune to changes in $\mathrm{BC}$ (Fig. $3 a, b$ ). The shape of ITD vectors for $\mathrm{BC}=1$, and those for nonzero BCs $(0.25,0.5$, and 0.75$)$, were highly correlated despite changes in their amplitude (Fig. 3c). The correlation between the ITD vectors for $\mathrm{BC}=0$ and 1 is expected to be low, because the ITD input is random for $\mathrm{BC}=0$.
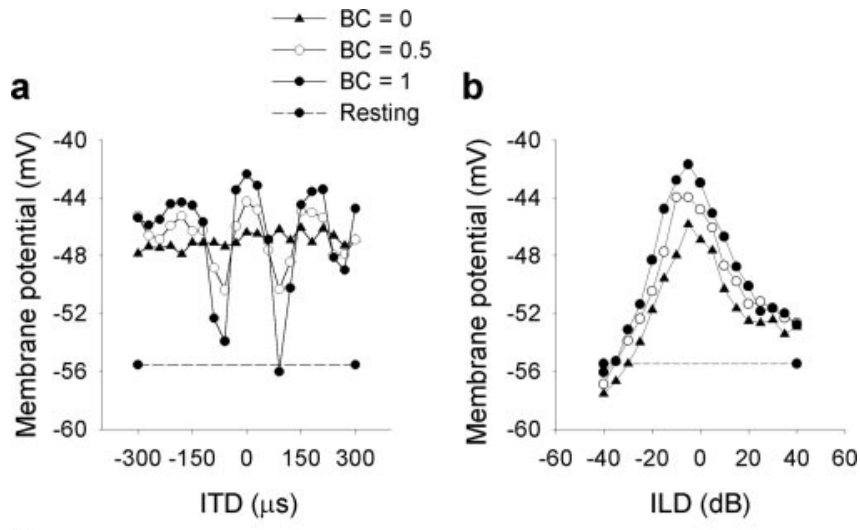

C

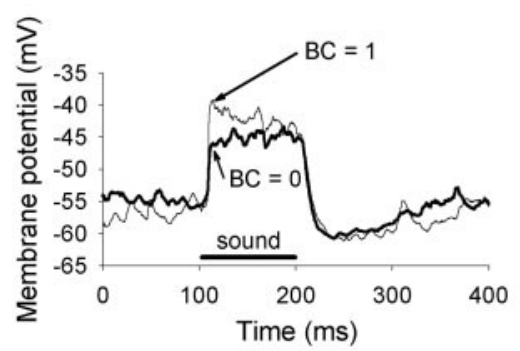

d

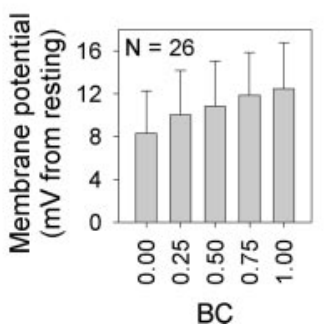

Figure 1. Binaural correlation and its effects on spatial tuning. The response of spacespecific neurons changes with BC. Overlaid ITD curves for PSPs $(a)$. Space-specific neurons were tuned to ILD despite changes in $B C(b)$. These data were obtained while the other parameter (ITD or ILD) was kept at the most favorable value for the neuron. Dashed lines represent mean resting potential. For $B C=0$, the membrane potential showed a smaller EPSP than for $B C=1$ and favorable ITD ( $c$ ). The amplitude of the depolarizing PSP decreased as BC became small ( $d$ ). Error bars are omitted for clarity in $a$ and $b$ and represent the SD in $d$. Traces were median filtered to eliminate spikes and averaged over five repetitions.

The random ITD vector caused by $\mathrm{BC}=0$ presents a special problem for the interpretation of SVD results, because multiplication and addition cannot be easily distinguished when the amplitude of one input becomes close to constant (Fig. 4). We examined which model, multiplicative or additive, produced matrices that matched the data matrices closer. Comparisons of the original matrices of PSP values with the matrices created by either multiplication of singular vectors obtained by SVD or by addition of vectors obtained by least-square fit of the data (see Materials and Methods) showed that the results of multiplication fit the data matrices better than those of addition (Fig. $3 d$ ). For all $\mathrm{BCs}$, the sums of squared differences were larger in the additive model than in the multiplicative one (sign test, $p<0.03$; Wilcoxon signed rank test, $p<0.005)$. Thus, even when the ITD input carries a significant amount of noise, it is not added but multiplied to the ILD input.

\section{Discussion}

We showed that the owl's space-specific neurons could perform multiplication of PSPs at all ITD input levels that could be created by varying BC. Because ICx neurons show little or no spiking response to $\mathrm{BC}=0$, even when the best ILD is combined with ITD, and because the sound levels used ( $40-50 \mathrm{~dB} \mathrm{SPL}$ ) were near the saturation level of the neurons that detect ITD (Albeck and Konishi, 1995; Peña et al., 1996), we were able to vary the ITD input to the ICx neurons over a significant part of their dynamic range. Thus, the multiplicative process works at all ITD input amplitudes that are known to result in spiking when combined with appropriate ILD input. Little is known about the biophysical 


\section{a}

Singular vectors

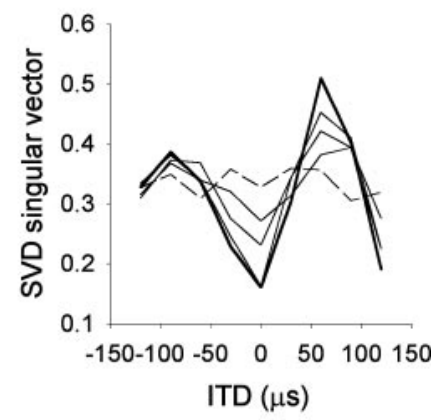

b
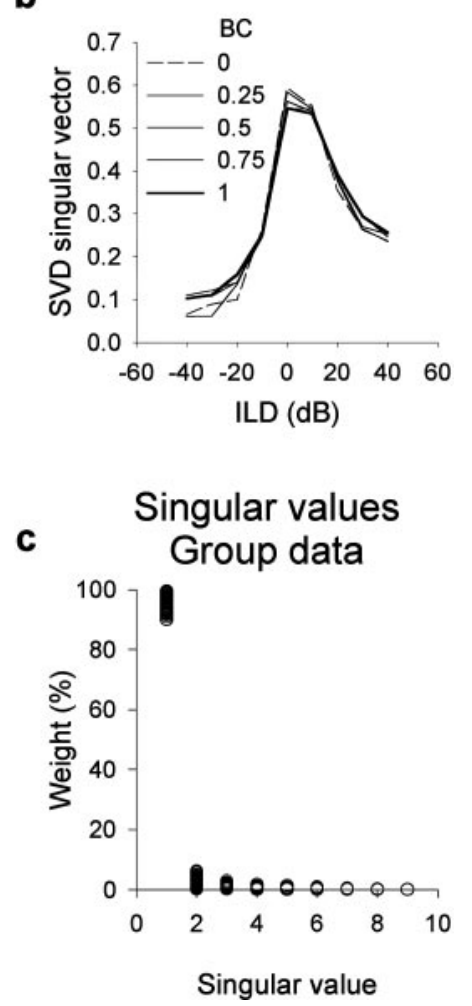

Figure 2. Multiplication of ITD and ILD as a function of binaural correlation. The singular value decomposition of subthreshold receptive fields obtained for five different $B C$ in the same neuron $(0,0.25,0.5,0.75$, and 1$)$ yielded different singular vectors for ITD $(a)$ but nearly constant singular vectors for ILD $(b)$. Despite $B C$ and in all the neurons, most of the weight was carried by the first singular value $(c)$, indicating that multiplication of two independent vectors applies in each case.

bases of multiplication in ICx neurons. The underlying mechanism combines PSPs of two different and independent origins in a nonlinear manner. GABA-mediated inhibition is known in ICx and contributes to the suppression of side peaks in ITD response curves (Fujita and Konishi, 1991; Mori, 1997). However, it is not known whether this inhibition plays any role in multiplication in ICX.

An independent and parallel processing of ITD and ILD was assumed for humans and demonstrated for barn owls (Stern and Colburn, 1978; Takahashi et al., 1984). The experiments performed on owls used a local anesthetic to silence or reduce the activity of relevant brain areas. The changes in the spike rate of ICx neurons in response to ITD and ILD served as the bases for judging the effects of inactivation. Partial inactivation of nucleus magnocellularis or nucleus angularis affected, respectively, only the ITD or ILD selectivity of ICx neurons. This finding led to the a

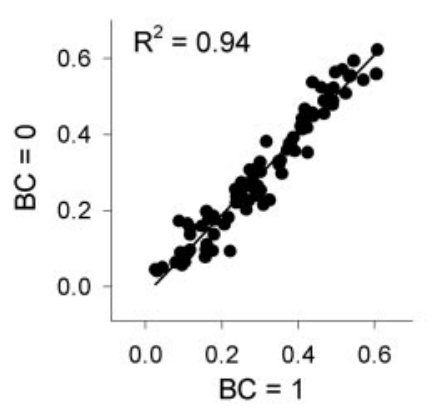

b

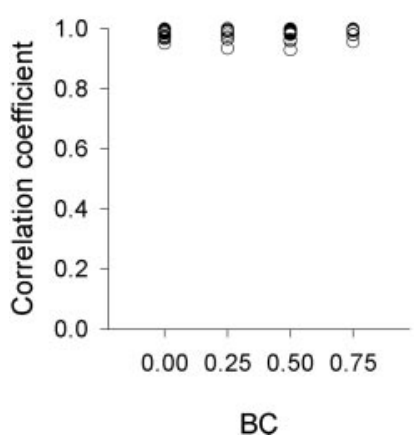

C

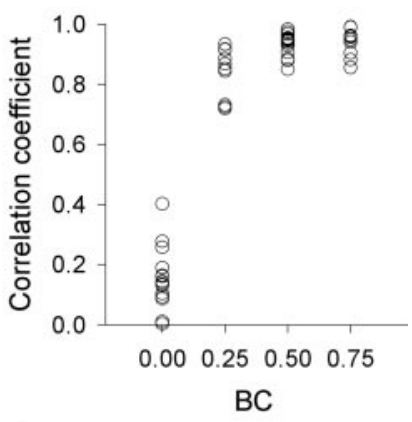

d

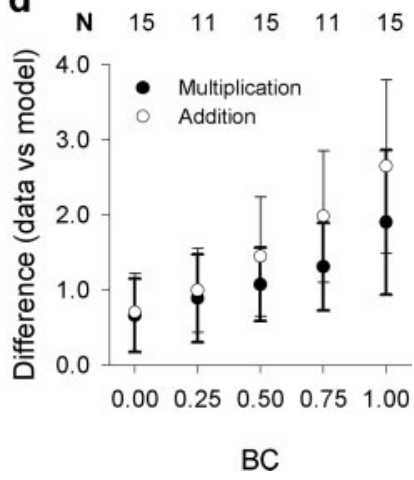

Figure 3. Performance of multiplicative and additive models. ILD singular vectors for $B C=$ 1 are plotted against ILD vectors for $B C=0$ to show their similarity in shape $(a)$. The solid line represents the linear regression and $R^{2}$ is the coefficient of determination. There is a high correlation between the ILD singular vector for $\mathrm{BC}=1$ and all lower $\mathrm{BCS}(b)$. The correlation between the ITD vector for $B C=1$ and $B C=0$, where the ITD input is reduced to noise, is low. However, ITD vectors for nonzero $B C$ s were highly correlated with that for $B C=1(c)$. The multiplicative model better fits the data than the additive model for those $B C s(d)$. The means of squared differences obtained by subtracting the additive (empty circles) and multiplicative (solid circles) models from the data matrices are plotted against BC. The number of neurons used in each case $(N)$ is indicated above the plot. Error bars represent SD.
Inputs

a

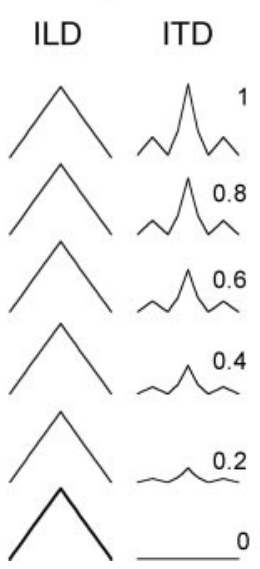

b

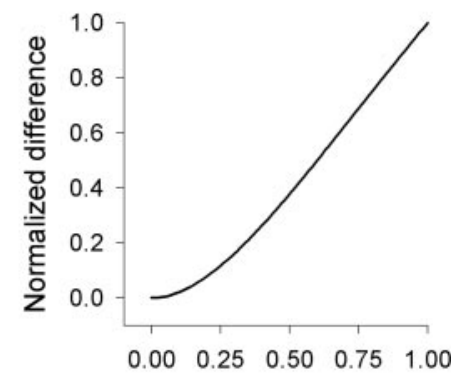

ITD input level
Figure 4. Convergence of multiplicative and additive models. We used model matrices to study the effects of reduced input on the differences between multiplication and addition ( $a$ ). We systematically scaled the amplitude of the ITD vector by numbers ranging from 0 to 1 (as indicated next to each plot). We performed SVD on a set of matrices created by addition of the ILD input to each of the scaled ITD inputs. The difference between these matrices and those derived by multiplication of the first singular vectors computed by SVD decreased as the amplitude of the ITD input declined $(b)$. The normalized differences between the data and the model matrices are the sum of the squared differences obtained by the element-by-element subtraction of the two matrices and divided by the maximum value. 
idea of independent processing of ITD and ILD (Takahashi et al., 1984). Here we showed that the ITD and ILD components of postsynaptic potentials in ICx neurons are multiplied as independent vectors despite input levels. This finding not only confirmed the independence of ITD and ILD inputs to ICx neurons but also explained their ITD-ILD combination selectivity or AND gate property.

The effects of BC on sound localization have been studied in humans and barn owls (Jeffress et al., 1962; Saberi et al., 1998). After perceiving a broadband signal at an arbitrary internal location between the ears, human subjects were asked to center the perceived sound source at the midpoint between the ears by varying the timing of sound. The SD of mean localization errors remained unchanged until $\mathrm{BC}$ was set $\sim 0.2-0.3$, below which the errors increased sharply. A similar pattern of error distribution occurred in barn owls, which performed a similar task by localizing phantom sound sources created by earphone-delivered signals. Saberi et al. (1998) explained this relationship between BC and localization by detection-theoretic analyses of neural data. Neurons of the optic tectum (OT) of barn owls respond to both sound and visual stimuli (Knudsen, 1982). Their auditory responses are similar to those of ICx neurons. The lowest BC for which OT neurons still detected ITD was also $0.2-0.3$. In the present paper, the lowest BC for which ICx neurons showed nonflat ITD vector was also $\sim 0.25$. The multiplicative process worked at this level, sustaining the combination selectivity for ITD and ILD required for the bicoordinate representation of space in the owl's midbrain.

\section{References}

Albeck Y, Konishi M (1995) Responses of neurons in the auditory pathway of the barn owl to partially correlated binaural signals. J Neurophysiol 74:1689-1700.

Andersen RA, Snyder LH, Bradley DC, Xing J (1997) Multimodal representation of space in the posterior parietal cortex and its use in planning movements. Annu Rev Neurosci 20:303-330.

Carr CE, Konishi M (1990) A circuit for detection of interaural time differences in the brain stem of the barn owl. J Neurosci 10:3227-3246.

Chance FS, Abbott LF, Reyes AD (2002) Gain modulation from background synaptic input. Neuron 35:773-782.

Egelhaaf M, Borst A, Reichardt W (1989) Computational structure of a biological motion-detection system as revealed by local detector analysis in the fly's nervous-system. J Opt Soc Am A 6:1070-1087.

Fujita I, Konishi M (1991) The role of GABAergic inhibition in processing of interaural time difference in the owl's auditory system. J Neurosci 11:722-739.

Gabbiani F, Krapp HG, Koch C, Laurent G (2002) Multiplicative computation in a visual neuron sensitive to looming. Nature 420:320-324.

Hatsopoulos N, Gabbiani F, Laurent G (1995) Elementary computation of object approach by a wide-field visual neuron. Science 270:1000-1003.

Jeffress LA, Robinson DA (1962) Formulas for coefficient of interaural correlation for noise. J Acoust Soc Am 34:1658.

Jeffress LA, Blodgett HC, Deatherage BH (1962) Effect of interaural correlation on the precision of centering a noise. J Acoust Soc Am 32:1122-1123.

Knudsen EI (1982) Auditory and visual maps of space in the optic tectum of the owl. J Neurosci 2:1177-1194.

Koch C (1999) Biophysics of computation: information processing in single neurons. Oxford University Press: New York.

Licklider JCR (1959) Three auditory theories. In: Psychology: a study of a science (Koch S, ed), pp 41-144. New York: McGraw-Hill.

Moiseff A, Konishi M (1983) Binaural characteristics of units in the owl's brainstem auditory pathway: precursors of restricted spatial receptive fields. J Neurosci 3:2553-2562.

Mori K (1997) Across-frequency nonlinear inhibition by GABA in processing of interaural time difference. Hear Res 111:17-30.

Olson JF, Knudsen EI, Esterly SD (1989) Neural maps of interaural time and intensity differences in the optic tectum of the barn owl. J Neurosci 9:2591-2605.

Peña JL, Konishi M (2001) Auditory spatial receptive fields created by multiplication. Science 292:249-252.

Peña JL, Viete S, Albeck Y, Konishi M (1996) Tolerance to sound intensity of binaural coincidence detection in the nucleus laminaris of the owl. J Neurosci 16:7046-7054.

Poggio T (1990) A theory of how the brain might work. Cold Spring Harbor Symp Quant Biol 55: 899-910.

Pouget A, Sejnowski TJ (1997) Spatial transformations in the parietal cortex using basis functions. J Cogn Neurosci 9:222-237.

Saberi K, Takahashi Y, Konishi M, Albeck Y, Arthur BJ, Farahbod H (1998) Effects of interaural decorrelation on neural and behavioral detection of spatial cues. Neuron 21:789-798.

Salinas E, Abbott LF (1996) A model of multiplicative neural responses in parietal cortex. Proc Natl Acad Sci USA 93:11956-11961.

Stern RM, Colburn HS (1978) Theory of binaural interaction based on auditory-nerve data. IV. A model for subjective lateral position. J Acoust Soc Am 64:127-140.

Sun HJ, Frost BJ (1998) Computation of different optical variables of looming objects in pigeon nucleus rotundus neurons. Nat Neurosci 1:296-303.

Takahashi TT, Moiseff A, Konishi M (1984) Time and intensity cues are processed independently in the auditory system of the owl. J Neurosci 4:1781-1786.

Yin TC, Chan JC (1990) Interaural time sensitivity in the medial superior olive of the cat. J Neurophysiol 64:465-488. 